\title{
Three-body recombination into deep bound states in a Bose gas with large scattering length
}

\author{
Eric Braaten and H.-W. Hammer \\ Department of Physics, The Ohio State University, Columbus, OH 43210, USA
}

(June 4, 2001)

\begin{abstract}
An effective field theory for the three-body system with large scattering length $a$ is applied to three-body recombination into deep bound states in a Bose gas. The recombination constant $\alpha$ is calculated to first order in the short-distance interactions that allow the recombination. For $a<0$, the dimensionless combination $m \alpha /\left(\hbar a^{4}\right)$ is a periodic function of $\ln |a|$ that exhibits resonances at values of $a$ that differ by multiplicative factors of 22.7. This dramatic behavior should be observable near a Feshbach resonance when $a$ becomes large and negative.
\end{abstract}

One important factor limiting the achievable density in Bose-Einstein condensates (BEC's) of trapped atoms is the loss of atoms through 3-body recombination [1,2]. Such losses occur when three atoms scatter to form a molecular bound state and a third atom. The kinetic energy of the final-state particles allows them to escape from the trapping potential. This process provides a unique window on 3-body dynamics of cold atoms. In a BEC, 3-body loss rates could also reveal new collective mechanisms involving a molecular condensate [3].

The recombination event rate can be parametrized as $\nu_{r e c}=\alpha n^{3}$, where $\alpha$ is the recombination constant and $n$ the density of the gas. The calculation of $\alpha$ in general is a complicated problem, because it is sensitive to the detailed behavior of the interaction potential. If the $s$-wave scattering length $a$ is large compared to the size of the atoms, however, the problem simplifies. Assuming that $a$ is the only important length scale, dimensional analysis implies $\alpha=\mathcal{C} \hbar a^{4} / m$, where $m$ is the mass of the atoms and $\mathcal{C}$ is dimensionless. For $a>0$, there can be 3 -body recombination into a shallow $s$-wave state with binding energy $\hbar^{2} /\left(m a^{2}\right)$. For either sign of $a$, there can also be recombination into deep molecular bound states. The coefficient $\alpha$ can then be split into two contributions $\alpha_{S}$ and $\alpha_{D}$ coming from recombination into the shallow and deep bound states, respectively.

The 3-body recombination into the shallow bound state has been studied in Refs. [4 -7]. For the $s$-wave contribution, $\mathcal{C}$ is an oscillatory function of $\ln a$ :

$$
\alpha_{S} \approx 67.9 \cos ^{2}\left[s_{0} \ln \left(a \Lambda_{*}\right)+1.74\right] \frac{\hbar a^{4}}{m} \quad(a>0),
$$

where $s_{0} \approx 1.0064$ and $\Lambda_{*}$ is the 3-body parameter introduced in Ref. [7]. In the adiabatic hyperspherical approximation, the oscillatory behavior can be explained by interference 
between paths connecting two hyperspherical potentials [5,6]. Within the framework of effective field theory, this unique behavior is due to scaling violations created by a 3-body interaction required for consistent renormalization [7]. For $a<0$, there is no shallow bound state (there is a low-energy virtual state instead) and $\alpha_{S} \equiv 0$.

Effective field theory (EFT) is a powerful method for describing systems composed of particles with wave number $k$ much smaller than the inverse of the characteristic range $R$ of their interaction. EFT focuses on the universal long-distance aspects of the problem, by modelling the interactions as pointlike [8]. For wave numbers $k \ll 1 / R$, one can expand in powers of the small variable $k R$. Generically, the scattering length $a$ is comparable to $R$, and the expansion is effectively in powers of $k a$. In the case of large scattering length $a \gg R$, the dependence on $k a$ must be treated nonperturbatively. In the EFT, a single 3-body parameter is necessary and sufficient to renormalize the ultraviolet divergences at leading order in $k R$ [9]. The scattering length and this 3-body parameter describe all low-energy 3-body observables up to errors of order $R / a$.

In this Letter, we use EFT methods to study the recombination constant $\alpha_{D}$ for deep bound states. The deep bound states are outside the range of the effective theory and cannot be treated explicitly. Nevertheless, their contribution to 3-body recombination can be calculated within the EFT by using the optical theorem (cf. Fig. 1(a)). The 3-body

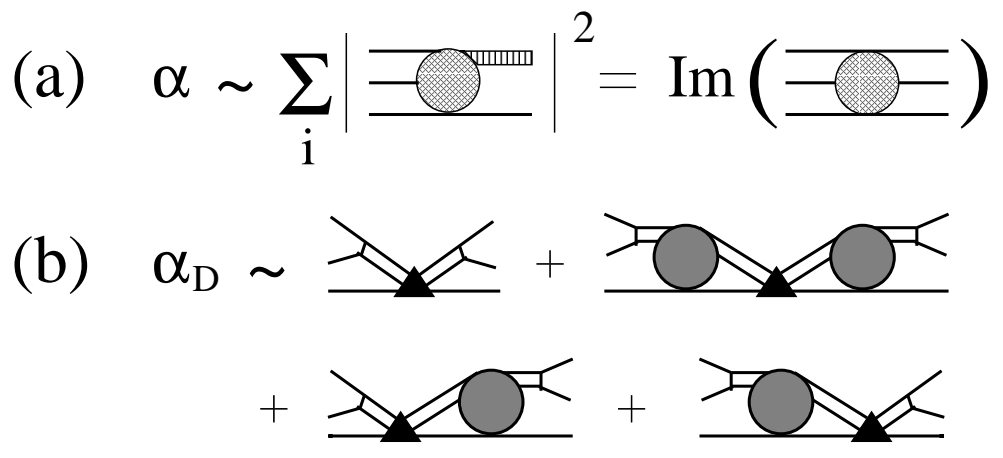

FIG. 1. (a) Illustration of the optical theorem. Two-body bound states are indicated by the shaded double line. (b) Diagrams for the contribution of the deep bound states. The single (double) lines represent the exact propagators for the field $\psi(d)$ and the triangle denotes an insertion of $i h^{\prime}$.

recombination channels generate imaginary parts in the $3 \rightarrow 3$ scattering amplitude. The imaginary part from recombination into the shallow bound state is dynamically generated in the EFT. The imaginary part from 3-body recombination into deep bound states can be taken into account by adding a local interaction term to the EFT.

A similar approach has been used successfully to describe the effects of the annihilation decays of positronium in QED and heavy quarkonium in QCD within the framework of nonrelativistic EFT's [10]. The annihilation of positronium into photons involves intermediate states with relativistic electrons and positrons that are outside the range of validity of the EFT. However, the effects of the annihilation process on $e^{+} e^{-}$-scattering at low energies can be taken into account systematically by adding local 4-fermion operators with imaginary coefficients to the effective Lagrangian. In the case of recombination into a molecule with binding energy $B \gg \hbar^{2} /\left(m a^{2}\right)$, the molecule and the recoiling atom will emerge with large 
momenta of order $\sqrt{m B}$ which is outside the domain of the EFT. Up to corrections of order $p^{2} /(m B)$, where $p$ is the scale of the momentum of the three atoms, the effect of the recombination process on the $3 \rightarrow 3$ scattering amplitude can be taken into account with a local 3-body interaction term whose coefficient has an imaginary part. The interaction is local, because the three atoms have to approach to within a distance of order $\hbar / \sqrt{m B} \ll a$ to recombine. The imaginary 3 -body coefficient accounts collectively for recombination into all deep bound states. It could in principle be calculated if the 2- and 3-body potentials describing the interactions between atoms were known with sufficient accuracy. In practice, it has to be determined from experimental data. Once this parameter is fixed, the dependence of $\alpha_{D}$ on $a$ and $\Lambda_{*}$ can be predicted.

In Ref. [7], we have calculated $\alpha_{S}$ for a large, positive scattering length $a$. In the following, we extend this work to calculate $\alpha_{D}$ for large $a$ of either sign. For simplicity, we now set $\hbar=1$. We start with a local Lagrangian for a nonrelativistic boson field $\psi$ and an auxiliary field $d$ with the quantum numbers of two bosons [7,9]:

$$
\begin{aligned}
\mathcal{L} & =\psi^{\dagger}\left(i \frac{\partial}{\partial t}+\frac{\vec{\nabla}^{2}}{2 m}\right) \psi+d^{\dagger} d \\
& -\frac{g}{\sqrt{2}}\left(d^{\dagger} \psi \psi+\psi^{\dagger} \psi^{\dagger} d\right)+\left(h+i h^{\prime}\right) d^{\dagger} d \psi^{\dagger} \psi+\ldots
\end{aligned}
$$

where $h^{\prime}$ is the free parameter accounting for recombination into the deep states. The dots denote terms with more derivatives and/or fields; those with more fields do not contribute to the 3-body amplitude, while those with more derivatives are suppressed at low energy. The couplings $g$ and $h$ in Eq. (2) can be eliminated in favor of $a$ and $\Lambda_{*}$, respectively. The atom propagator has the usual nonrelativistic form $i /\left(\omega-p^{2} / 2 m\right)$. The 2 -atom scattering amplitude for incoming atoms with momenta $\pm \vec{k}$ has the form $(-1 / a-i k)^{-1}$. The exact propagator for $d$ has a pole at $\omega=-1 /\left(m a^{2}\right)+\vec{p}^{2} /(4 m)$ corresponding to the shallow bound state $[7,9]$.

Before describing the calculation of $\alpha_{D}$, we summarize the calculation of $\alpha_{S}$ in Ref. [7]. The coefficient for recombination into the shallow bound state can be expressed as $\alpha_{S}=$ $512 \pi^{2}\left|t\left(p_{f}\right)\right|^{2} /(\sqrt{3} m)$, where $t(p)$ is the amplitude for the transition between three atoms at rest and a final state consisting of an atom and a shallow $s$-wave state with momentum $p$ in their center-of-momentum frame. This amplitude is evaluated on shell at the value $p_{f}=2 /(\sqrt{3} a)$ prescribed by energy conservation. However, $t(p)$ is also defined at off-shell values of $p$ as the solution of an integral equation that contains the 2 - and 3 -body interaction terms [7]. Note that $t$ is related to the amplitude $T$ of Ref. [7] via $t(p)=m T(p) /\left(48 \pi^{3 / 2} \sqrt{a}\right)$. The integral equation is regularized by a momentum cutoff $\Lambda$. All observables can be made independent of the cutoff by adjusting the 3-body coefficient $h(\Lambda)$ as a function of $\Lambda$. The evolution of $h$ with $\Lambda$ approaches a limit cycle: $\Lambda^{2} h$ varies periodically between $+\infty$ and $-\infty$ as $\Lambda$ increases by multiplicative factors of $\exp \left(\pi / s_{0}\right) \approx 22.7$ [9]. Since all observables are cutoff independent, we can choose a $\Lambda$ for which $h(\Lambda)=0$. The 3-body parameter $\Lambda_{*}$ then appears in the upper limit of the integral and we obtain a renormalized equation [1],

$$
t(p)=\frac{2}{p^{2}}+\frac{2}{\pi} \int_{0}^{\Lambda_{n}} \frac{d q q^{2} t(q)}{-1 / a+\sqrt{3} q / 2-i \epsilon}
$$




$$
\times \frac{1}{p q} \ln \left|\frac{q^{2}+p q+p^{2}}{q^{2}-q p+p^{2}}\right|
$$

with $\Lambda_{n}=\Lambda_{*} \exp \left[\left(n \pi+\arctan \left(1 / s_{0}\right)\right) / s_{0}\right]$ and $s_{0} \approx 1.0064$. The integer $n$ should be large enough that errors of $\mathcal{O}\left(1 /\left(\Lambda_{n} a\right)\right)$ can be neglected. In practice, one can often choose $n=1$. Solving the integral equation for $t(p)$ and evaluating the solution as described above, we obtain the result in Eq. (1). The integral equation (3) also has a solution for $a<0$, but there is no shallow bound state in this case and $\alpha_{S}=0$.

In order to describe the recombination into the deep bound states, we use the optical theorem as discussed above and include an imaginary part $h^{\prime}$ in the coefficient of the 3body contact interaction. We assume that the $h^{\prime}$ term can be treated as a perturbation at first order. However, the external legs have to be dressed with initial and final state interactions to all orders. We have to calculate the diagrams shown in Fig. 1(b), where the blob represents the solution of Eq. (3) and the triangle represents an insertion of $i h^{\prime}$. This leads to

$$
\alpha_{D}=\frac{288 \pi^{2} a^{2}}{m} \frac{h_{1}}{\Lambda_{n}^{2}}\left|1+\frac{2}{\pi} \int_{0}^{\Lambda_{n}} \frac{d q q^{2} t(q)}{-1 / a+\sqrt{3} q / 2-i \epsilon}\right|^{2},
$$

where we have defined $h^{\prime} /\left(m g^{2}\right)=h_{1} / \Lambda_{n}^{2}$. Eq. (4) gives a renormalized expression for $\alpha_{D}$ up to corrections of $\mathcal{O}\left(1 /\left(\Lambda_{n} a\right)\right)$. Since $t(q)$ is known numerically from solving Eq. (3), the calculation of $\alpha_{D}$ entails the numerical evaluation of the integral in Eq. (4).

There are now two cases to consider: $a>0$ and $a<0$. We first consider the case $a>0$. Evaluating Eq. (4), we find that the amplitude is dominated by a linear divergence in the integral and is proportional to $\Lambda_{n} a$. The factors of $\Lambda_{n}$ cancel and the result is proportional to $a^{4}$ with a coefficient that, within the numerical accuracy, is independent of $\Lambda_{*} a$ :

$$
\alpha_{D}=0.041 h_{1} \frac{\hbar a^{4}}{m} \quad(a>0)
$$

In Fig. 2(a), we show both $\alpha_{S}$ and $\alpha_{D}$ for $a>0$, with $h_{1}$ adjusted such that $\alpha_{D}=10$. The recombination into the deep bound states fills in the zeroes of $\alpha_{S}$, so that they become at best local minima of the recombination rate.

We now turn to the more interesting case $a<0$. Evaluating Eq. (雨), we find that the amplitude is again dominated by a linear divergence in the integral. However, the coefficient of $a^{4} / m$ is not a pure number but exhibits scaling violations. $\alpha_{D}$ has the remarkable dependence on $\Lambda_{*}|a|$ shown in Fig. 2(b). The coefficient diverges at a sequence of values of $\Lambda_{*}|a|$ that are equally spaced on a logarithmic scale. To a high numerical accuracy, the results can be described by the formula

$$
\alpha_{D}=\frac{5.61 h_{1}}{\sin ^{2}\left[s_{0} \ln \left(\Lambda_{*}|a|\right)+1.77\right]} \frac{\hbar a^{4}}{m} \quad(a<0) .
$$

The oscillatory dependence on $\ln |a|$ is in accord with a general scaling law by Efimov [12]. There are divergences whenever $s_{0} \ln \left(\Lambda_{*}|a|\right)+1.77$ is an integer multiple of $\pi$. They occur because a 3-body Efimov state [12] has been tuned to threshold by the variation of $a$. The divergences are artifacts of the first order perturbation theory in $h_{1}$, which treats the Efimov 

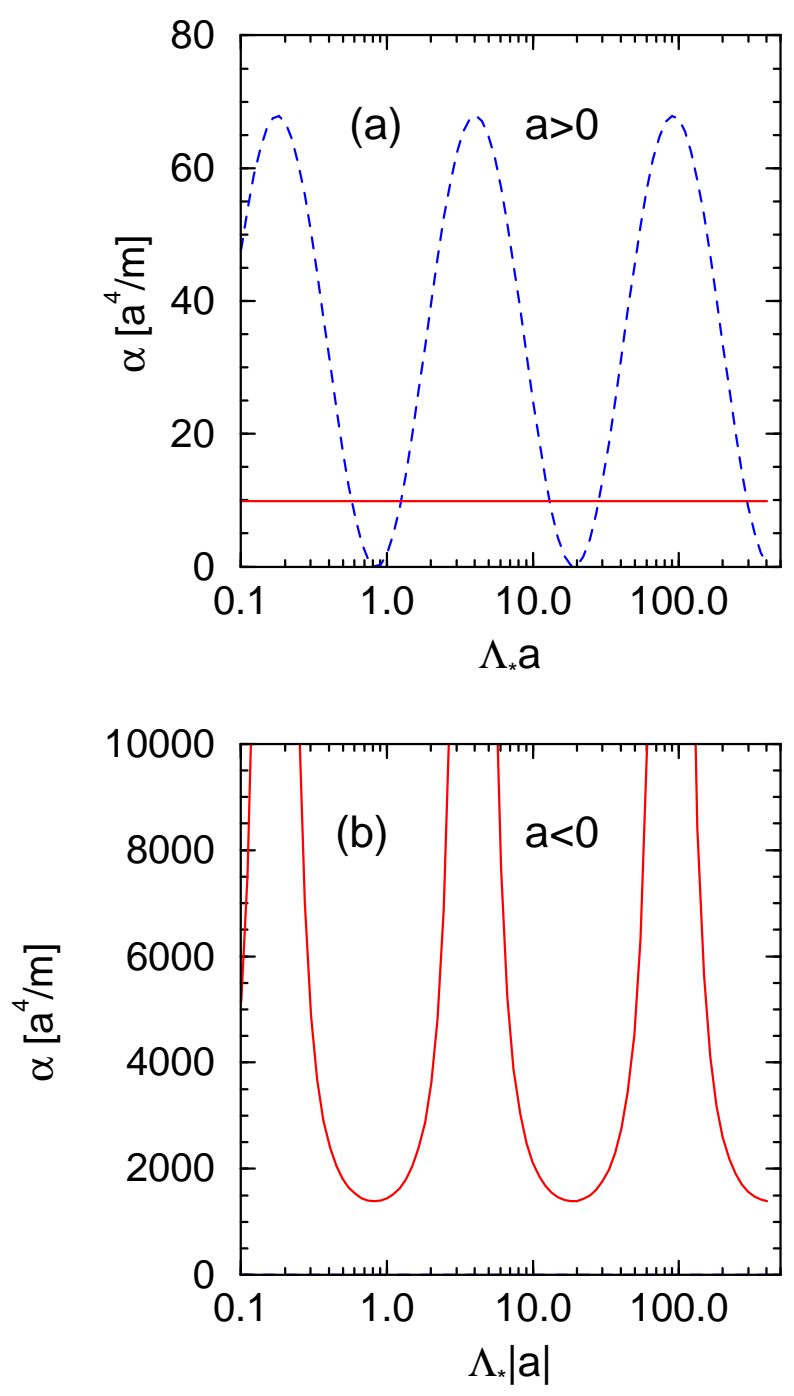

FIG. 2. The recombination coefficients $\alpha_{D}$ (solid line) and $\alpha_{S}$ (dashed line) as functions of $|a| \Lambda_{*}$ for $a>0$ and $a<0$. The free parameter $h_{1}$ in $\alpha_{D}$ was chosen such that $\alpha_{D}=10$ for $a>0$.

states below the 3-body threshold as having sharp energies. However, these Efimov states aquire a width that depends on $h_{1}$ from recombination into the deep states [13]. This will change the divergences into resonances at which the recombination rate is enhanced by a factor $\left(\hbar^{2} / \Gamma m a^{2}\right)^{2}$, where $\Gamma$ is the width of an Efimov state at the 3-body threshold. First-order perturbation theory in $h_{1}$ is justified if $\Gamma \ll \hbar^{2} /\left(m a^{2}\right)$, except within $\Gamma$ of a resonance. The determination of the height of the resonance peaks requires a calculation that is nonperturbative in $h_{1}$. Note that the elastic 3-body scattering amplitude will also exhibit these resonances if $a<0$. For $a>0$, an Efimov state at the 3-body threshold has a width of order $\hbar^{2} /\left(m a^{2}\right)$ from recombination into the shallow 2-body bound state. Consequently, there should be no resonances in the recombination rate.

The EFT results for $\alpha$ depend on two parameters $\Lambda_{*}$ and $h_{1}$ that are determined by interatomic interactions at distances much smaller than $|a|$. They have predictive power only if $a$ can be varied as a function of some external parameter and if the dependence of 
$\Lambda_{*}$ and $h_{1}$ on that parameter is known. One case in which there is predictive power is near a Feshbach resonance, where $a$ is varied by tuning a molecular bound state close to the threshold of two atoms by using an external magnetic field $B$. Short-distance parameters must be smooth functions of $B$. Over the narrow interval of a Feshbach resonance, they can be approximated by a linear or even constant function of $B$. Linear dependence of the short-distance parameter $g$ in (2) corresponds to $a(B)=a_{0}+\Delta /\left(B-B_{0}\right)$, where $a_{0}$ is the off-resonant scattering length and $\Delta$ is the width of the Feshbach resonance. The short distance parameters $\Lambda_{*}$ and $h_{1}$ can be approximated by constants near the resonance at $B_{0}$.

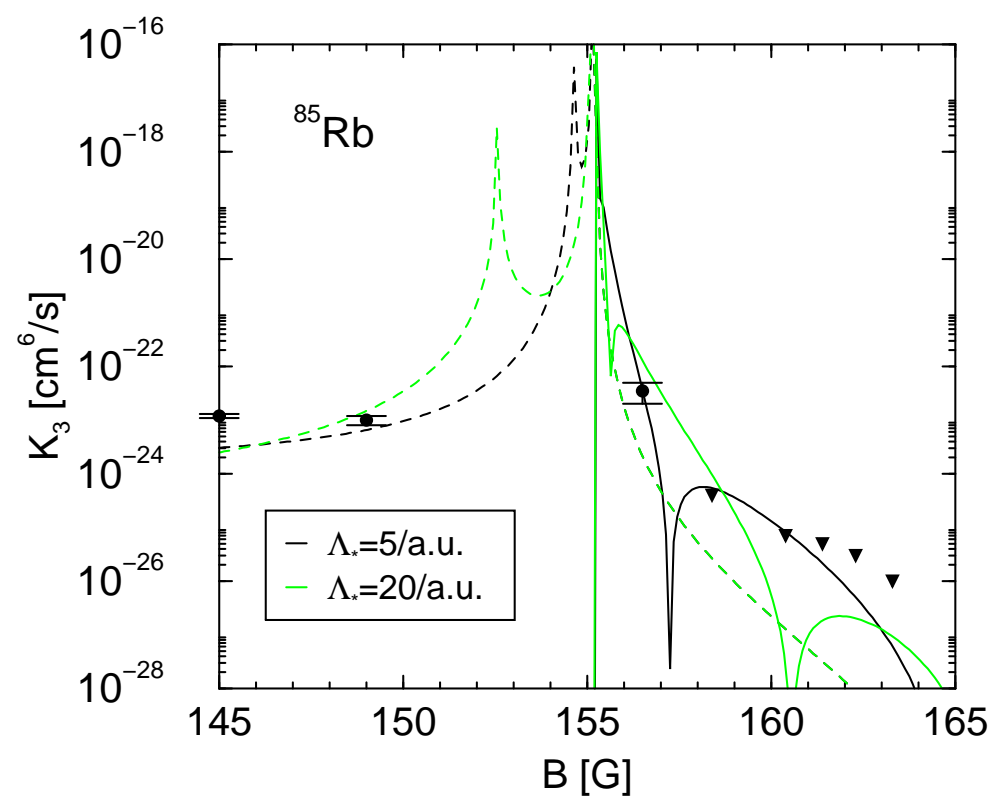

FIG. 3. The atom loss rate $K_{3}=3 \alpha$ for a gas of ultracold ${ }^{85} \mathrm{Rb}$-atoms. The dots (triangles) represent data (upper bounds) for $K_{3}$ from Ref. [2]. The EFT predictions for $K_{3 S}$ (solid lines) and $K_{3 D}$ (dashed lines) are shown for $\Lambda_{*}=5 /$ a.u. (black curves) and $\Lambda_{*}=20 /$ a.u. (grey curves). The arbitrary normalization factor in $K_{3 D}$ is set to $h_{1}=24.7$.

In Fig. 3 we compare our theory with measurements of the atom loss rate coefficient $K_{3}=3 \alpha$ in an ultracold gas of ${ }^{85} \mathrm{Rb}$-atoms by Roberts et al. [2]. In ${ }^{85} \mathrm{Rb}$, there is a Feshbach resonance with width $\Delta=11.5 \mathrm{G}$ at a magnetic field $B_{0}=155.2 \mathrm{G}$ [2]. In Fig. [3, the scattering length is negative below the Feshbach resonance at $B_{0}=155.2 \mathrm{G}$ and positive above. Consequently, the shallow bound state contributes to $K_{3}$ only above the resonance. The free parameter $h_{1}=24.7$ has been fixed to reproduce the overall magnitude of $K_{3}$ below the resonance. For $a>0$, this value of $h_{1}$ leads to a contribution of the deep bound states to the loss rate $\left(K_{3 D}\right)$ that is much smaller than the contribution of the shallow bound state $\left(K_{3 S}\right)$ except near the minima of $K_{3 S}$. One might be able to determine $h_{1}$ experimentally from the depth of these minima. In Fig. 目, we have plotted $K_{3 D}$ and $K_{3 S}$ for two values of the 3-body parameter: $\Lambda_{*}=5 /$ a.u. and $\Lambda_{*}=20 /$ a.u.. The parameter $\Lambda_{*}$ would be determined very accurately if a local minimum or maximum in the loss rate were observed.

Divergences in the 3-body recombination rate for $a<0$ have been observed previously by Esry et al. [6]. They calculated $\alpha$ numerically for various 2-body potentials, most of them with a single bound state (either shallow or deep). They varied $a$ from large negative values 
to large positive values by tuning the depth of the potential. For $a>0, \alpha / a^{4}$ increases to a maximum at some value $a_{\max }$ and then decreases to an interference minimum as predicted by Eq. (11). For $a<0, \alpha / a^{4}$ diverges at some value $a_{\text {div }}$. The ratio $\left|a_{\text {div }}\right| / a_{\text {max }}$ ranged from 1.6 to 2 for the potentials studied. Since the maxima of $\alpha_{S} / a^{4}$ and the divergences of $\alpha_{D} / a^{4}$ in Fig. 2 appear at the same values of $|a|$, the EFT predicts $\left|a_{\operatorname{div}}\right| / a_{\max }=1$. The discrepancy between the results of Ref. [6] and the EFT prediction can be explained by a variation in the effective short-distance parameters $\Lambda_{*}$ and $h_{1}$ as the depth of the potential is tuned to vary $a$. Near a Feshbach resonance, the short distance behavior of the potential is essentially unchanged as $B$ is varied. Thus $\Lambda_{*}$ and $h_{1}$ should be nearly constant and the EFT prediction should hold as long as $|a|$ is much larger than its off-resonance value.

We have shown that the EFT approach to the 3-body system with large scattering length can be applied to recombination into deep bound states. For $a>0$, this contribution fills in the interference minima of the recombination rate into shallow bound states, transforming them into local minima of the total recombination rate. For $a<0$, the EFT approach makes the dramatic prediction that the recombination rate for zero-momentum atoms should exhibit resonances due to Efimov states at a sequence of values for $a$ that differ by multiplicative factors of $\exp \left(\pi / s_{0}\right) \approx 22.7$. Provided the widths of these Efimov states are numerically small compared to $\hbar^{2} /\left(m a^{2}\right)$, the resonances should be observable as sharp peaks in the recombination rate near a Feshbach resonance.

We thank C.H. Greene and J.P. Burke for useful discussions and for providing us with numerical results of their calculations. This research was supported by NSF grant PHY9800964 and DOE grant DE-FG02-91-ER4069. 


\section{REFERENCES}

[1] J. Söding et al., Appl. Phys. A 69, 257 (1999); J. Stenger et al., Phys. Rev. Lett. 82, 2422 (1999).

[2] J.L. Roberts, N.R. Claussen, S.L. Cornish, and C.E. Wieman, Phys. Rev. Lett. 85, 728 (2000).

[3] F.A. van Abeelen and B.J. Verhaar, Phys. Rev. Lett. 83, 1550 (1999); V. Yurowsky et al., Phys. Rev. A 60, R765 (1999); E. Timmermans et al., ccond-mat/9805323].

[4] P.O. Fedichev, M.W. Reynolds, and G.V. Shlyapnikov, Phys. Rev. Lett. 77, 2921 (1996).

[5] E. Nielsen and J.H. Macek, Phys. Rev. Lett. 83, 1566 (1999).

[6] B.D. Esry, C.H. Greene, and J.P. Burke, Phys. Rev. Lett. 83, 1751 (1999).

[7] P.F. Bedaque, E. Braaten, and H.-W. Hammer, Phys. Rev. Lett. 85, 908 (2000).

[8] G.P. Lepage, in From Actions to Answers, TASI'89, ed. T. DeGrand and D. Toussaint, (World Scientific, Singapore, 1990); D.B. Kaplan, [nucl-th/9506035].

[9] P.F. Bedaque, H.-W. Hammer, and U. van Kolck, Nucl. Phys. A 646, 444 (1999); Phys. Rev. Lett. 82, 463 (1999).

[10] G.T. Bodwin, E. Braaten, and G.P. Lepage, Phys. Rev. D 51, 1125 (1995).

[11] H.-W. Hammer and T. Mehen, Nucl. Phys. A (in print) [nucl-th/0011024].

[12] V. Efimov, Sov. J. Nucl. Phys. 12, 589 (1971); 29, 546 (1979).

[13] E. Nielsen and B.D. Esry, Bull. Am. Phys. Soc 45, 67 (2000); V. Efimov, in Few Body Dynamics, A.N. Mitra et.al. (eds.), North Holland, Amsterdam, 1976. 